\title{
Synthesis of uniform porous Fe micro-flakes with excellent magnetic properties in $\mathrm{GHz}$ range
}

\author{
Yanhui $\mathrm{Wu}^{1,2, a}$, Mangui Han ${ }^{1,2, \mathrm{~b}}$ and Longjiang Deng ${ }^{1,2, \mathrm{c}}$ \\ ${ }^{1}$ National Engineering Research Center of Electromagnetic Radiation Control Materials, University \\ of Electronic Science and Technology of China, Chengdu 610054, China; \\ ${ }^{2}$ State Key Laboratory of Electronic Thin Films \& Integrated Devices, University of Electronic \\ Science and Technology of China, Chengdu, 610054, China.
}

aYanhui153@outlook.com, b magnet@uestc.edu.cn, 'brillice153wyh@126.com

Keywords: Fe micro-flakes, porous structure, shape anisortropy, permeability.

\begin{abstract}
Porous Fe micro-flakes with uniform size and well-defined shape have been successfully synthesized by hydrogen thermal reduction of the $\alpha-\mathrm{Fe}_{2} \mathrm{O}_{3}$ precursor flakes. The thicknesses and planar sizes of those porous flakes fall into the range of 208-375nm and 10-23 $\mu \mathrm{m}$, respectively. Static magnetic properties and high frequency permeability of the porous Fe micro-flakes have been charactered. The porous Fe micro-flakes with large saturation magnetization and small coercivity values have also show high real and imaginary permeability values. The two wide magnetic loss peaks could be owing to the magnetic anisortropy dispersion which is caused by the porous structure. What's more, it implies that the composites with porous Fe microflakes can be used for the purpose of electromagnetic noise attenuation over a wide frequency range.
\end{abstract}

\section{Introduction}

Many physical and chemical properties of particles in nano-scale and micro-scale, such as electronic, magnetic, optical, catalytic properties, strongly depend on sizes and shapes. Therefore, obtaining those particles with controllable sizes and shapes has attracted great attention [1, 2]. There are many methods to prepare those particles, for instance, ball milling, solution method, vapor method and so on [3]. Two dimension structures have low weight, large surface area, specific facets and more active sites when comparing to zero dimension and one dimension structures [4].

Recently, with the rapid development of wireless communication technologies in gigahertz $(\mathrm{GHz})$ range, such as mobile telephones, intelligent transports and satellite broadcast systems, the accompanying electromagnetic interferences (EMI) are increasingly serious [5, 6]. Hence, the electromagnetic wave absorption materials are promising to solve this problem. Generally speaking, the electromagnetic wave absorption materials are divided into two types: dielectric materials and magnetic materials. Dielectric materials achieve absorption though dielectric loss which is caused by electric polarization, while magnetic materials that is mainly though magnetic loss related to dynamic magnetization process [7]. Ferromagnetic metal particles have attracted much more attention than ferrite due to the higher permeability and higher operating frequency [8]. Particularly, two dimension ferromagnetic metal particles have extended snoek's law which is owing to the strong shape anisortropy but still retained high permeability in $\mathrm{GHz}$ range [5]. Thus, flake-like ferromagnetic metal particles should realize excellent electromagnetic wave absorption properties.

In general, for the metal flakes prepared by milling, the sizes are discrete and the shapes are irregular. However, the metal flakes with uniform size and shape can be prepared by solution method. But, since the high reduction potential, Fe flakes can be hardly obtained by liquid reduction process [9]. Fortunately, iron oxide can be prepared by solution method [10]. And then, Fe flakes can be obtained by reducing the precursor by hydrogen [11].

In this contribution, we intend to prepare plate-like $\alpha-\mathrm{Fe}_{2} \mathrm{O}_{3}$ by hydrothermal approach. Then the precursor has been reduced by hydrogen annealing to acquire the porous flaky Fe. The porous Fe 
flake not only can be uniform size and shape, but also can be a candidate in absorbing the electromagnetic noise.

\section{Experiments}

In this study, the quality of all chemicals used is in the analytical grade and used without further purification. $\alpha-\mathrm{Fe}_{2} \mathrm{O}_{3}$ micro-flakes were synthesized by a facile hydrothermal route. In the fabrication process, $60 \mathrm{~mL}$ distilled water were divided equally with the serial number " $A$ " and " $B$ ". Subsequently, $0.05 \mathrm{~mol} \mathrm{Fe}\left(\mathrm{NO}_{3}\right)_{3}=9 \mathrm{H}_{2} \mathrm{O}$ and $0.45 \mathrm{~mol} \mathrm{NaOH}$ were dissolved in " $\mathrm{A}$ " and " $\mathrm{B}$ " to form two homogeneous solutions, respectively. Solution "A" and "B" were mixed rapidly with vigorous stirring for nearly 10 minutes. The mixture was placed into a $100 \mathrm{~mL}$ Teflon-lined autoclave and then hydrothermally treated at $473 \mathrm{~K}$ for $10 \mathrm{~h}$ and cooled down to room temperature. The collected powder products were washed several times in deionized water and industrial alcohol, and then were dried at $333 \mathrm{~K}$ for 12 hours.

Porous Fe micro-flakes were prepared by hydrogen-thermal reduction. $\alpha$ - $\mathrm{Fe}_{2} \mathrm{O}_{3}$ micro-flakes were reduced in hydrogen atmosphere at $773 \mathrm{~K}$ for $1 \mathrm{~h}$. The reduced products were cooled in $\mathrm{N}_{2}$ atmosphere to room temperature naturally in the furnace.

A scanning electron microscope (SEM, JEOL, JSM 7600F) was used to observe and analyze the morphologies of the collected powder sample. The dimensions (length, width and thickness) of ribbons were measured by the software named "Smile View ${ }^{\circledR}$ ". X-ray diffraction (SHIMADZU, XRD 7000) was used to analyze the phase structures and a hysteresis loop has been taken by a vibrating sample magnetometer (VSM) to characterize room temperature static magnetic properties for the particles. To investigate the microwave performance, a vector network analyzer (Agilent 8720ET) was used to measure $\mu$ in $0.5-10 \mathrm{GHz}$ by inserting a toroidal shape sample into the coaxial waveguide. The toroidal sample was a mixture of particles and wax. And the volume fraction of the particles is $18.34 \%$. The inner diameter, outer diameter and thickness of the samples are 3.0, 7.0 and $3 \mathrm{~mm}$, respectively.

\section{Results and Discussions}
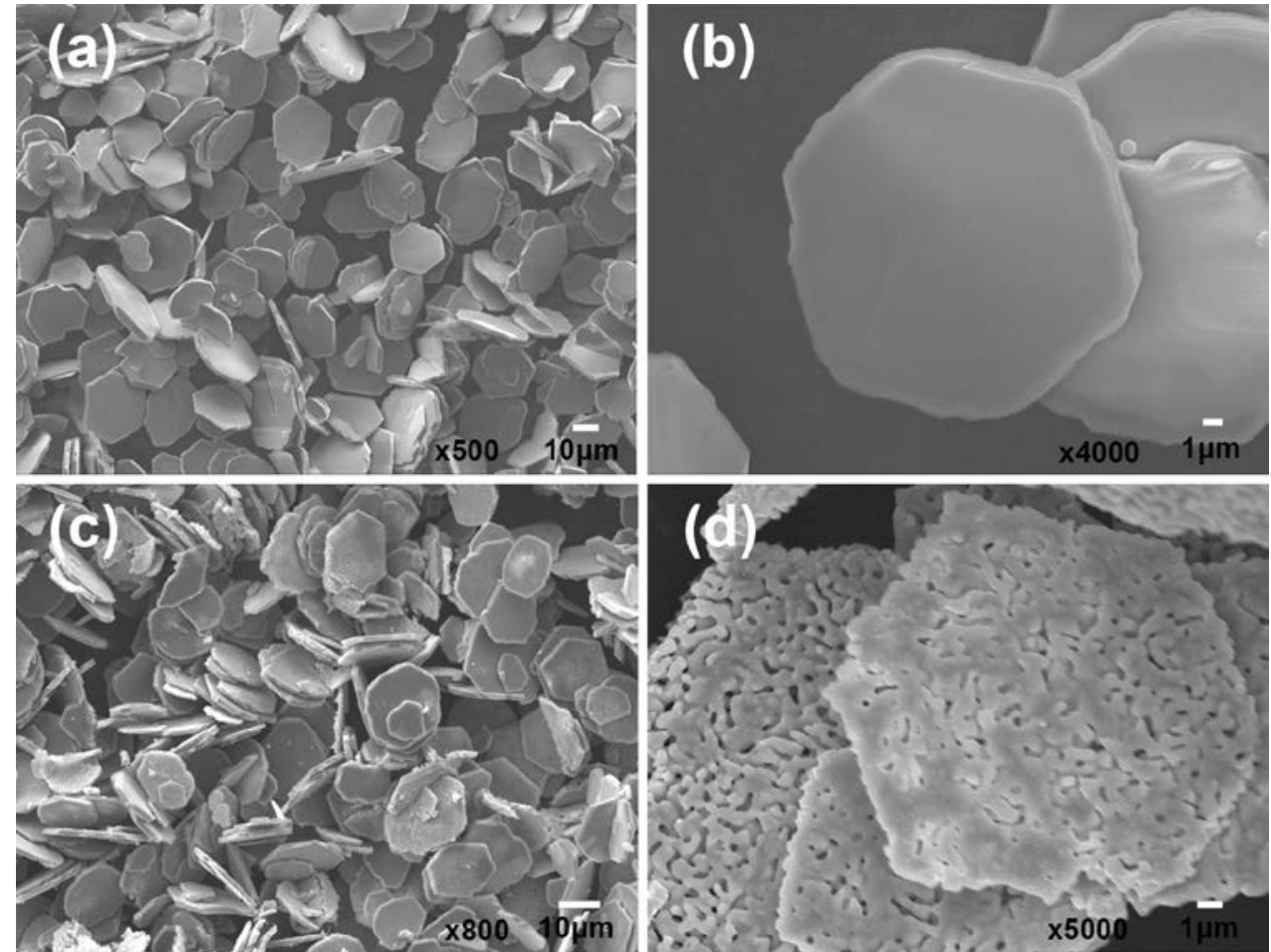

Fig. 1 SEM images of (a) $\alpha-\mathrm{Fe}_{2} \mathrm{O}_{3}$ precursor. (b) partial enlarged detail of (a). (c) reduced porous $\mathrm{Fe}$ flakes. (d) partial enlarged detail of (c). 
The morphologies of the prepared precursor and the $\mathrm{H}_{2}$ annealed particles are shown in Fig. 1 (a, b) and Fig. 1(c, d), respectively. The planar sizes of both particles mostly fall into the range of 10-23 $\mu \mathrm{m}$. And the thicknesses mostly fall into the range of 208-375nm. Obviously, the surfaces of the precursors are smooth, after $\mathrm{H}_{2}$ annealing, many porous with bore diameter about 100-200 nm formed on the surfaces. After annealing, the surface area increases while the apparent density decreases. In addition, according to the width and thickness values, the aspect ratio is larger than 10 which could caused strong shape anisortropy.

The XRD patterns of the precursor and the $\mathrm{H}_{2}$ annealed particles are shown in Fig. 2. The crystal structure for the particles of each type is a single pure phase. For the precursor, most of the diffraction peaks which are marked by the rhombus are related to the $\alpha-\mathrm{Fe}_{2} \mathrm{O}_{3}$ phase (JCPDS No. 33-0664), while for the $\mathrm{H}_{2}$ annealed particles, the several trilateral-marked peaks are related to $\alpha$-Fe phase (JCPDS No. 05-0696). According to the Scherrer's equation [12], the grain sizes of $\alpha-\mathrm{Fe}_{2} \mathrm{O}_{3}$ and $\alpha-\mathrm{Fe}$ phase are $386.3 \mathrm{~nm}$ and $160.1 \mathrm{~nm}$, respectively.

As seen from the hysteresis loop in Fig. 3(a), the $\alpha-\mathrm{Fe}_{2} \mathrm{O}_{3}$ precursor is magnetic. the magnetization is very small but the coercivity $\left(H_{c}\right)$ values gets a very high value, 1293.4 Oe. Seen from Fig. 3(b), the saturation magnetization $\left(\sigma_{s}\right)$ and coercivity $\left(H_{c}\right)$ values of the porous Fe flakes are $224 \mathrm{emu} / \mathrm{g}$ and 53 Oe, respectively. The results indicate that the porous Fe flakes have good soft magnetic properties. So much high saturation magnetization value would lead to large permeability values. Besides, the variations of coercivity values could be owing to the different grain size of the each magnetic phase [13].

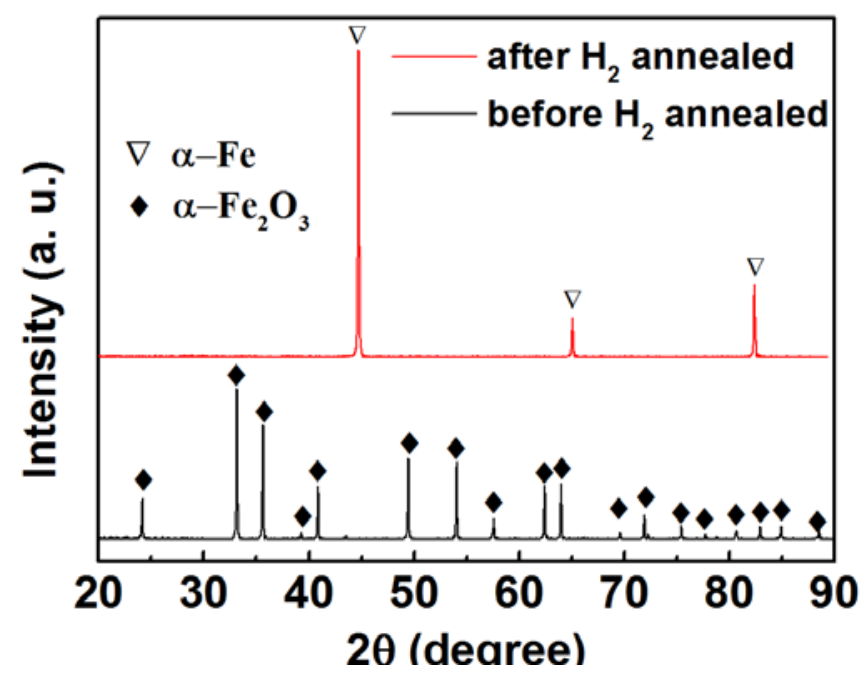

Fig. 2 XRD patterns of $\alpha-\mathrm{Fe}_{2} \mathrm{O}_{3}$ precursor before and after $\mathrm{H}_{2}$ annealed.
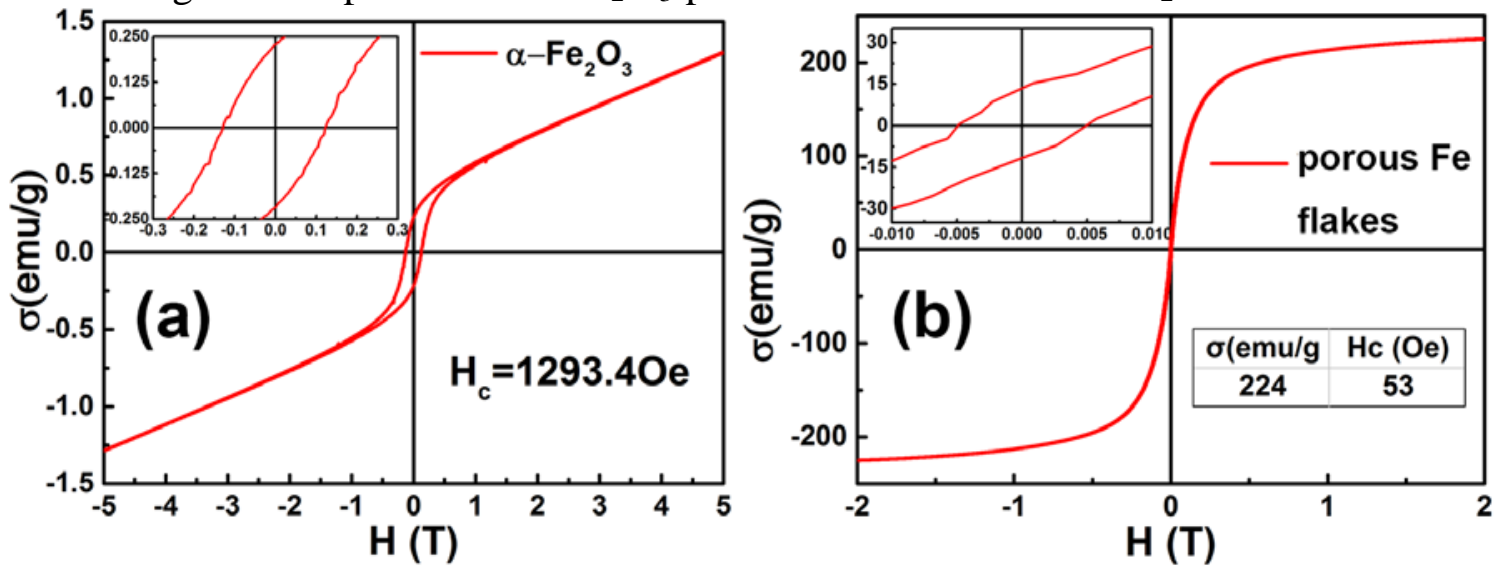

Fig. 3 The magnetic hysteresis loops of (a) $\alpha-\mathrm{Fe}_{2} \mathrm{O}_{3}$ precursor. (b) porous Fe flakes. The insets are the enlarged zone of the loops and the listed $\sigma_{s}$ and $H_{c}$ values.

The measured microwave permeability spectra of composites containing Fe microflakes are shown in Fig. 4. The real part $\left(\mu_{s}^{\prime}\right)$ and imaginary part $\left(\mu_{s}^{\prime \prime}\right)$ of effective permeability at $0.5 \mathrm{GHz}$ are about 3.5 and 1.5, respectively. The frequency dependence of imaginary part of permeability reveals a 
superposition of two resonance peaks. Generally speaking, the resonance peaks are related to nature resonance in $\mathrm{GHz}$ range [6]. And according to our previous work [7], the origins of wide magnetic loss peaks is that the inhomogeneity of composites system will result in the distribution of magnetic anisotropy fields, which will give rise to the distribution of natural resonances of spins rotation. In this case, the porous structure causes inhomogeneity of the shape anisortropy. Therefore, the two wide magnetic loss peaks could be understood. Moreover, it implies that the composites with porous Fe microflakes can be used for the purpose of electromagnetic noise attenuation over a wide frequency range.

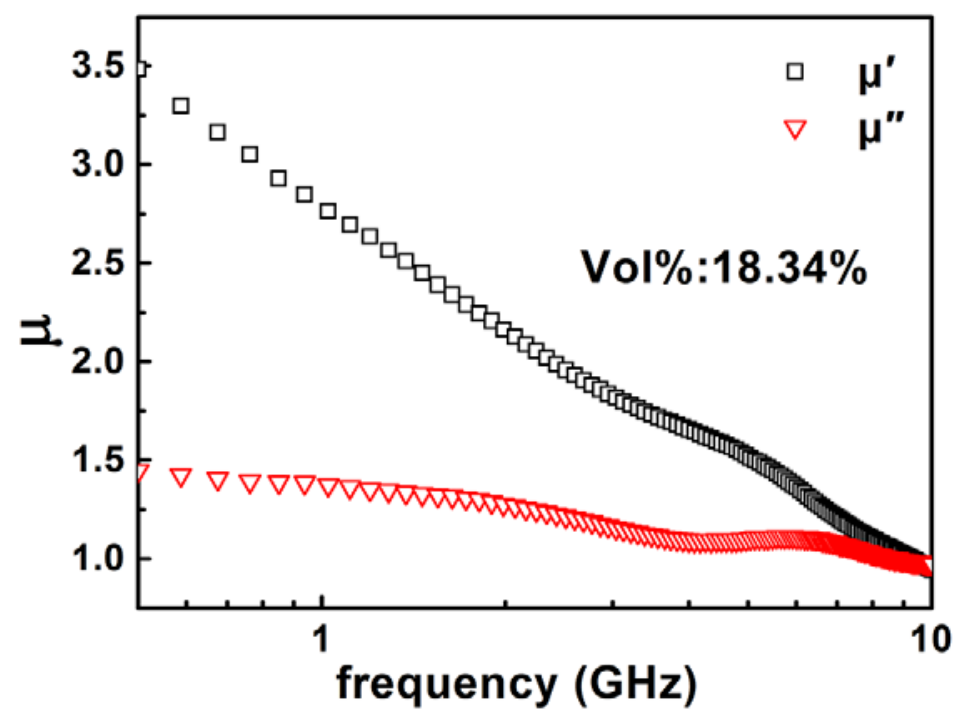

Fig. 4 permeability of the composites containing the porous Fe flakes. The insert is the volume fraction of the porous Fe flakes in the composites.

\section{Acknowledgment}

This work is funded by the National Natural Science Foundation of China (Grant No. 61271039), and the scientific projects of Sichuan province (2013JQ0006, 2015HH0016). It is also supported by the Open Foundation of National Engineering Research Center of Electromagnetic Radiation Control Materials (ZYGX2014K003-4).

\section{References}

[1] Y. G. Sun and Y. N. Xia, Shape-controlled synthesis of gold and silver nanoparticles, Science, 298 (2002) 2176-2179.

[2] J. N. Anker, W. P. Hall, O. Lyandres, N. C. Shah, J. Zhao and R. P. Van Duyne, Biosensing with plasmonic nanosensors, Nat. Mater., 7 (2008) 442-453.

[3] V. F. Puntes, K. M. Krishnan and A. P. Alivisatos, Colloidal nanocrystal shape and size control: the case of cobalt, Science, 291 (2001) 2115-2117.

[4] S. J. Guo and S. J. Dong, Graphene nanosheet: synthesis, molecular engineering, thin film, hybrids, and energy and analytical applications, Chem. Soc. Rev., 40 (2011) 2644-2672.

[5] Y. H. Wu, M. G. Han, Z. K. Tang, and L. J. Deng, Eddy current effect on the microwave permeability of Fe-based nanocrystalline flakes with different sizes, J. Appl. Phys., 115 (2014) 163902.

[6] L. B. Kong, Z. W. Li, L. Liu, R. Huang, M. Abshinova, Z. H. Yang,C. B. Tang, P. K. Tan, C. R. Deng and S. Matitsine, Recent progress in some composite materials and structures for specific electromagnetic applications, Inter. Mater. Rev., 58 (2013) 203-259. 
[7] Y. H. Wu, M. G. Han, Z. K. Tang, and L. J. Deng, Studies on the microwave permittivity and electromagnetic wave absorption properties of Fe-based nano-composite flakes in different sizes, J. Appl. Phys., 118 (2015) 023902.

[8] L. D. Liu, Y. P. Duan, S. H. Liu, L. Y. Chen, and J. B. Guo, J. Magn. Magn. Mater. 322 (2010) 1736.

[9] J. G. Li, Y. Qin, X. L. Kou and J. J. Huang, Microstructure and Magnetic Properties of FexNi1-x Alloy Nanoplatelets, J. Nanosci. Nanotechnol., 5 (2005) 1699-1706.

[10] M. Tadic, N. Citakovic, M. Panjan, Z. Stojanovic, D. Markovic and V. Spasojevic, Synthesis, morphology, microstructure and magnetic properties of hematite submicron particles, J. Alloys Compd., 509 (2011) 7639-7644.

[11] G. B. Sun, B. X. Dong, M. H. Cao, B. Q. Wei and C. W. Hu, Hierarchical dendrite-like magnetic materials of $\mathrm{Fe} 3 \mathrm{O} 4, \gamma-\mathrm{Fe} 2 \mathrm{O} 3$, and Fe with high performance of microwave absorption, Chem. Mat., 23 (2011) 1587-1593.

[12] U. Holzwarth and N. Gibson, The Scherrer equation versus the 'Debye-Scherrer equation', Nature Nanotechnology, 6 (2011) 534.

[13] Y. Yoshizawa, in Handbook of Advanced Magnetic Materials, edited by D. J. Sellmyer, Y. Liu, and D. Shindo (Tsinghua University Press, Beijing, 2005), Vol. IV, Chap. 4, p. 124. 